\title{
Dapagliflozin as an Adjunct Therapy to Insulin in Patients with Type 1 Diabetes Mellitus: Efficacy and Safety of this Combination
}

Johan H Jendle, ${ }^{1}$ Francisco J Ampudia-Blasco, ${ }^{2}$ Martin Füchtenbusch ${ }^{3}$ and Paolo Pozzilli ${ }^{4}$

1. Institution of Medical Sciences, Örebro University, Örebro, Sweden; 2. Endocrinology and Nutrition Department, Clinic University Hospital Valencia, INCLIVA Biomedical Research Institute, Valencia, Spain; 3. Diabetes Centre at Marienplatz, Munich, Germany, Diabetes Research Study Group e.V. at Helmholt Zentrum Munich, Germany; 4. University Campus Bio-Medico, Rome, Italy

$\mathrm{T}$ he prevalence of type 1 diabetes (T1D) is increasing worldwide. T1D reduces life expectancy due to complications including cardiovascular disease. Sodium-glucose co-transporter (SGLT) inhibitors are a new class of drugs developed to treat type 2 diabetes (T2D), and now they can be used as an adjunct to insulin in T1D. In clinical trials, they have been shown to improve glycaemic control and decrease body weight without the risk of increased hypoglycaemia and with a reduction in insulin dose. Four SGLT2 inhibitors have been approved in Europe for the treatment of T2D, while only dapagliflozin and sotagliflozin, a dual SGLT1 and SGLT2 inhibitor approved in 2019, have been approved for the treatment of T1D. Both can be used as an adjunct therapy in combination with insulin in adults with a body mass index (BMI) of $\geq 27 \mathrm{~kg} / \mathrm{m}^{2}$, inadequately controlled with insulin. In Europe, dapagliflozin is the only currently available SGLT2 inhibitor indcated as adjunct therapy for patients with T1D. The subgroup of patients with a BMI of $\geq 27 \mathrm{~kg} / \mathrm{m}^{2}$ from the DEPICT-1 and -2 trials (Dapagliflozin Evaluation in Patients with Inadequately Controlled Type 1 diabetes) showed similar reduction in hyperglycaemia and body weight but no significant increased risk of diabetic ketoacidosis (DKA) than the overall trial population. The risk of DKA has been shown to increase in patients with T1D treated with adjunct therapy with SGLT2 inhibitors, and studies on sotagliflozin and empagliflozin have suggested a dose response. Thus, it is important to educate patients and doctors how to recognize symptoms of upcoming DKA and mitigate it. An independent DKA education programme has recently been developed to instruct patients with T1D being treated with SGLT inhibitor therapies with and without insulin pumps to prevent, identify and treat DKA. Despite these considerations, clinical trials support the use of SGLT2 inhibitors in the management of T1D. The benefits and potential risks of dapagliflozin as an adjunct therapy to insulin in adults with T1D should be considered in each individual case. Here we discuss the efficacy and safety of dapagliflozin as adjunct therapy in patients with T1D.

\section{Keywords}

Dapagliflozin, sodium glucose co-transporter, type 1 diabetes, adjunctive therapy, insulin

Disclosures: Johan H Jendle serves on advisory boards for AstraZeneca, Boehringer Ingelheim, Eli Lilly, Medtronic, Merck, NovoNordisk and Sanofi; speakers bureau for AstraZeneca, Boehringer Ingelheim, Eli Lilly, Medtronic, NovoNordisk and Sanofi; and has received grants to institution from Medtronic and NovoNordisk. Francisco J Ampudia-Blasco has served on advisory panels for Abbott, AstraZeneca, Boehringer Ingelheim, Eli Lilly, GlaxoSmithKline, LifeScan, Medtronic, Merck, Novartis, NovoNordisk, Pfizer, Roche and Sanofi; and has received research support from Abbott, Astrazeneca, Boehringer Ingelheim, Bayer, Eli Lilly, GlaxoSmithKline, Lifescan, Merck, NovoNordisk, Pfizer, Sanofi and Servier. Martin Füchtenbusch serves on advisory boards for AstraZeneca, Boehringer Ingelheim, Eli Lilly and Berlin Chemie; and is a speaker for AstraZeneca, Boehringer Ingelheim, Eli Lilly, Berlin Chemie, Abbott, Sanofi, MSD and NovoNordisk. Paolo Pozzilli serves on advisory boards for AstraZeneca and Sanofi; speakers bureau for Eli Lilly, Sanofi and AstraZeneca; and has received grants to institution from Sanofi, Eli Lilly, AstraZeneca, Medtronic, GSK, Augen and NovoNordisk. Acknowledgement: Medical writing support, including preparation of the drafts under the guidance of the authors, was provided by Ray Ashton of Touch Medical Communications and was funded by AstraZeneca.

Review Process: Double-blind peer review.

Compliance with ethics: This article involves a review of the literature and did not involve any studies with human or animal subjects performed by any of the authors.

Authorship: The named authors meet the International Committee of Medical Journal Editors (ICMJE) criteria for authorship of this manuscript, take responsibility for the integrity of the work as a whole, and have given final approval for the version to be published.

Access: This article is freely accessible at touchENDOCRINOLOGY.com (C) Touch Medical Media 2021.

Received: 15 May 2020

Accepted: 10 August 2020

Published online: 26 March 2021

Citation: touchREVIEWS in Endocrinology. 2021;17(1):12-20

Corresponding author: Johan H Jendle, Institution of Medical Sciences, Örebro University, S-701 82 Örebro, Sweden. E: Johan.Jendle@oru.se

Support: The publication of this article was supported by AstraZeneca. The views and opinions expressed

in the article are those of the authors and not necessarily those of AstraZeneca. 
The prevalence of diabetes type 1 (T1D) and type 2 (T2D) is increasing worldwide; the number of people with diabetes was 463 million in 2019, but it is estimated to reach 700 million by $2045 .{ }^{1}$ The incidence of T1D is increasing in most countries. ${ }^{1}$ Age- and sex-standardized incidence rates from 1998 to 2013 (up to 25 years) from 26 European centres (European Diabetes [EURODIAB] registry) in 22 countries have been published. Individuals were aged 0-14 years and had been newly diagnosed with T1D. Incidence increased significantly in all centres (except for those in Ireland and Marche, Italy). Poland reported the highest increase (6.6\% per year). The pooled analysis from all centres indicated a 3.4\% (95\% confidence interval [Cl]: 2.8-3.9\%) rise in incidence rate annually, although some centres in high-incidence countries reported reduced rates of increase in the last few years. ${ }^{2}$ Data from the Swedish Childhood Diabetes Register indicated that the yearly incidence of T1D showed a greater increase in younger people compared with older age groups. ${ }^{3}$

Despite the benefits of improved glycaemic control in T1D, many patients are unable to achieve or maintain glycaemic targets. However, to reduce the treatment burden associated with intensive insulin therapy, adjunctive therapies to T1D have been evaluated. Sodium-glucose co-transporter-2 (SGLT2) inhibitors are a new class of oral therapy for T2D that have been more recently evaluated in T1D as well. Phase III trials with dapagliflozin in the DEPICT (Dapagliflozin Evaluation in Patients with Inadequately Controlled Type 1 diabetes) trials, empagliflozin in the EASE (Empagliflozin as Adjunctive to insulin thErapy) trial, and sotagliflozin (a dual SGLT1 and SGLT2 inhibitor) in the inTandem trials (in combination with insulin) have been performed in adults with T1D. ${ }^{4-8}$

\section{Unmet needs in type 1 diabetes}

The main unmet needs in T1D therapy include reduction of glycated haemoglobin (HbA1C) without increasing hypoglycaemia event rate, reduction of glucose variability, reduction of excess body weight, lowering acute and long-term diabetic complications and mortality. 10,11

\section{Insufficient glycaemic control}

An assessment of glycaemic control in people with T1D from 18 countries $(n=324,501)$ found a considerable variation. For example, in those aged $\geq 25$ years old, only $20.5-53.6 \%$ had an $\mathrm{HbA} 1 \mathrm{c}$ of $<9.4 \%(58.5 \mathrm{mmol} / \mathrm{mol}){ }^{12}$ Similarly, data from 25,833 adults and children, collected by the T1D Exchange Clinical Registry in the USA, showed that, on average, HbA1C was $8.3 \%(67.2 \mathrm{mmol} / \mathrm{mol})$, with the highest $\mathrm{HbA} 1 \mathrm{c}$ seen in those aged 13-25 years. ${ }^{13}$ European data from Sweden and Denmark indicate slightly lower average HbA1c levels than in the USA, but a significant proportion of patients are still missing their individual goals. In Sweden, the youngest patients (18-21 years) with T1D reported the highest HbA1C levels, but although this value increased from 2009 to 2012 from 8.3\% to $8.5 \%$ (67.4 $\mathrm{mmol} / \mathrm{mol}$ to $69.1 \mathrm{mmol} / \mathrm{mol}$ ) it decreased in 2013 to $8.3 \%$ (67.6 mmol/mol). ${ }^{14}$ A study from France found that $24.3 \%$ of 3,858 patients with T1D in the SAGE (Study of Adults' Glycemia in T1D) trial achieved the target of $<7.0 \% \mathrm{HbA} 1 \mathrm{C}(53.0 \mathrm{mmol} / \mathrm{mol})$, with the average being $8.0 \%(63.4 \mathrm{mmol} / \mathrm{mol}) .^{15}$

\section{Unacceptable high risk of hypoglycaemia}

Hypoglycaemia, particularly severe hypoglycaemia, and its consequences are one of the most limiting factors to improve glycaemic control in T1D. This is a major acute complication of T1D that can cause seizures and loss of consciousness. Severe hypoglycaemia is relatively common in patients with T1D, occurring in $11.8 \%$ of adults in the USA T1D Exchange Clinical Register (12-month frequency); this increases with rising and lowering HbA1C levels. ${ }^{16}$ However, severe hypoglycaemia has also not been associated with increased risk of both cardiovascular disease nor mortality in subjects with T1D. ${ }^{17}$

As mentioned previously, glucose variability over time may better predict hypoglycaemia in patients with diabetes than HbA1C levels. 18,19 In a recent study, Gerbaud et al. showed that glycaemic variability above $2.7 \mathrm{mmol} / \mathrm{L}(48.7 \mathrm{mg} / \mathrm{dL})$ was the strongest independent predictor for mid-term major adverse cardiovascular events (MACE) in people with diabetes and acute coronary syndrome..$^{20}$ In addition, a 2016 study in 1,706 adolescents with T1D showed that glycaemic variation was associated with retinopathy, albuminuria, and cardiac autonomic neuropathy. ${ }^{21}$ Data from France in patients with T1D demonstrated that, compared with mean glucose level, short-term glycaemic variability results in more hypoglycaemia than glucose alone when the glucose threshold is $3.0 \mathrm{mmol} / \mathrm{L}(54 \mathrm{mg} / \mathrm{dL}) .^{22}$ Recently, an international consensus report on continuous glucose monitoring gave guidance for time in range as a metric of glycaemic control, providing more actionable information than HbA1c alone. ${ }^{23}$

\section{Excessive weight gain with intensive insulin therapy}

Weight gain commonly occurs with intensive insulin therapy over time and is a considerable problem in patients with T1D; in many cases it is associated with high blood pressure and some lipid abnormalities. ${ }^{10,11}$ In the Pittsburgh Epidemiology of Diabetes Complications study, patients were initially assessed between 2004 and 2007 and followed for 18 years. After 18 years, there was a $47.0 \%$ increase in patients with T1D becoming overweight and a seven-fold increase in obesity versus baseline. ${ }^{10}$

\section{Prevention of cardiovascular complications in type 1 diabetes}

Cardiovascular complications in T1D were evaluated in the Diabetes Control and Complications Trial (DCCT) and Epidemiology of Diabetes Interventions and Complications (EDIC) study. Intensive insulin treatment reduced the number of any cardiovascular events compared with conventional treatment ( 46 events in 31 patients versus 98 events in 52 patients, respectively; $\mathrm{p}=0.02$ ) but weight increased with intensive treatment. ${ }^{10,24}$ An assessment of quartiles of weight gain in the two treatment groups indicated that the intensive treatment group had higher proportional weight gain (body mass index [BMI] medium change 6.1 $\left.\mathrm{kg} / \mathrm{m}^{2}\right)$. In addition, patients on intensive treatment had an increased use of drugs for both hypertension and lipids compared with quartiles 1-3 and the equivalent conventional treatment quartiles. Despite this, during the follow-up of 26 years, the incidence of major and total cardiovascular events was similar. ${ }^{25}$ Nevertheless, those who gained excessive weight in EDIC (Q4 intensive treatment group) had a revascularization rate that was nearly twice as high compared with the Q1-3 INT (minimal weight gain) groups. Thus, excessive weight gain in T1D is associated with higher event rates indicative of atherosclerosis, albeit not MACE. ${ }^{24}$

Some phenotypes and age at diagnosis of patients with T1D may be linked to an increased risk of cardiovascular events. ${ }^{26}$ Lithovius et al. suggested that data-driven metabolic subtypes can predict outcomes in patients with T1D. In particular, the metabolic syndrome subtype is predictive of cardiovascular death (standard metabolic rate: 11.0 in men and 23.4 in women; $p<0.001$ ). ${ }^{27}$ Furthermore, findings from the Swedish National Diabetes Register indicated that cardiovascular outcomes and survival can be predicted by age at onset of T1D, with the greatest excess risk occurring in women. Thus, cardio-protection should be considered in all age groups, but particularly in early onset T1D. ${ }^{28}$ 


\section{Further reduction of microvascular complications} Results from the DCCT and EDIC study comparing intensive insulin treatment ( $\geq 3$ daily insulin injections or using an insulin pump) with conventional therapy demonstrated that the onset of diabetic retinopathy was delayed, progression slowed and the cumulative incidence of persistent microalbuminuria was reduced in the intensive treatment groups. ${ }^{29,30}$ In the DCCT, patients who had a higher estimated time in range (70-180 mg/dL, 3.9-10 mmol/L) had a lower frequency of microvascular complications. ${ }^{31}$

\section{Reduced life expectancy in type 1 diabetes}

A prospective study of 24,691 people with T1D aged $\geq 20$ years evaluated life expectancy compared with a control group. ${ }^{32}$ In men with T1D at 20 years of age, life expectancy was reduced by 11.1 years (95\% Cl: 10.1-12.1), while in women the estimated loss was 12.9 years (95\% Cl: 11.7-14.1). Ischaemic heart disease accounted for the largest overall percentage reduction in life expectancy (36\% and 31\% in men and women, respectively) while diabetic coma or diabetic ketoacidosis (DKA) represented the highest percentage before the age of 50 years (29.4\% in men and $21.7 \%$ in women). ${ }^{31}$

\section{Sodium-glucose co-transporter inhibitors in the treatment of diabetes}

SGLT1 and SGLT2 are responsible for glucose re-absorption at different tissues. This occurs in the proximal tubules of the kidneys (for SGLT2 and to a lesser degree SGLT1) and small intestine (mainly SGLT1). ${ }^{33}$ Generally, SGLT2 re-absorbs approximately $90 \%$ of filtered glucose by active transport. ${ }^{34}$ Further along the proximal tubule in segments $2-3$, the remaining $10 \%$ of filtered glucose is re-absorbed by SGLT1. Expression of SGLT2 is very low in other tissues, whereas SGLT1 is expressed in many tissues including the small intestine, skeletal muscle, liver, lung, kidney and heart. ${ }^{32}$

Inhibition of SGLT2 transporters results in increased glucosuria and natriuresis, with reduction in $\mathrm{HbA} 1 \mathrm{C}$, body weight and blood pressure. ${ }^{35}$ Inhibition of SGLT1 transporters is associated with blunted intestinal glucose absorption and decreased postprandial hyperglycaemia. ${ }^{32-34}$ Currently, four SGLT2 inhibitors are approved in Europe for the treatment of T2D: dapagliflozin, canagliflozin, empagliflozin and ertugliflozin. As mentioned previously, dapagliflozin $5 \mathrm{mg}$ received a positive Committee for Medicinal Products for Human Use (CHMP) opinion and was approved for adjunct therapy in combination with insulin in the EU (for adults with a BMI of $\geq 27 \mathrm{~kg} / \mathrm{m}^{2}$ ) and Japan in 2019 for T1D. Sotagliflozin, a dual SGLT2 and SGLT1 inhibitor, was approved in the EU in April 2019 (for adults with a BMl of $\left.\geq 27 \mathrm{~kg} / \mathrm{m}^{2}\right){ }^{36,37}$ In patients with T2D, SGLT2 inhibitors reduce $\mathrm{HbA} 1 \mathrm{c}$, fasting and postprandial blood glucose levels, body weight and blood pressure, and have also been shown to improve cardiovascular and renal outcomes. These effects are related to increasing urine glucose excretion, which results in lowering of blood glucose without raising the risk of hypoglycaemia independently of the remaining insulin secretion. ${ }^{38,39}$ In patients with T1D, they reduce HDA1C, body weight and blood pressure. Data from the DEPICT trials have shown that those with a BMl of $\geq 27 \mathrm{~kg} / \mathrm{m}^{2}$ benefit most, with a better benefit-risk ratio from treatment with dapagliflozin (see benefit-risk analysis in the subgroup of patients with a BMl of $\geq 27 \mathrm{~kg} / \mathrm{m}^{2}$ in DEPICT- 1 and -2$) .{ }^{40}$

Focusing on dapagliflozin, a real-world study of patients with T2D has shown efficacy both as monotherapy and in combination with other oral anti-diabetes therapies and insulin. ${ }^{41}$ In T2D, it is administered as a $10 \mathrm{mg}$ dose, except in those with hepatic impairment where a $5 \mathrm{mg}$ dose should be considered. A number of meta-analyses of randomized, controlled trials have evaluated the efficacy of dapagliflozin in approximately 4,000 patients with T2D. These meta-analyses indicate that the drug improves $\mathrm{HbA} 1 \mathrm{c}$ by an average of $0.5 \%$, fasting glucose by $1.1 \mathrm{mmol} / \mathrm{L}(19.8 \mathrm{mg} / \mathrm{dL}$ ), reduces weight by $2.0 \mathrm{~kg}$ or $4.5 \mathrm{~kg}$ (in combination with metformin and a sulphonylurea) and improves systolic/diastolic blood pressure by $4 / 2$ mmHg over $24-52$ weeks. ${ }^{42}$

The benefits in cardiovascular outcome and renal function seen for SGLT2 inhibitors in T2D have not been studied in T1D, but since many of the benefits are seen in patients without prevalent cardiovascular disease, it cannot be ruled out that they may also benefit patients with T1D. The DECLARE TIMI-58 (Dapagliflozin Effect on Cardiovascular Events) clinical trial enrolled 17,160 patients with T2D and established atherosclerotic cardiovascular disease or who had multiple risk factors for atherosclerotic cardiovascular disease. Treatment with dapagliflozin resulted in a lower rate of cardiovascular death or hospitalization for heart failure versus placebo (4.9\% versus 5.8\%; hazard ratio [HR]: 0.83 ; 95\% Cl: 0.73-0.95; $p=0.005)$. The cumulative incidence of the renal composite outcome was reduced in the dapagliflozin group versus placebo. A renal event was recorded in $4.3 \%$ of patients treated with dapagliflozin compared with $5.6 \%$ in the placebo group (HR: 0.76; $95 \%$ $\mathrm{Cl}: 0.67-0.87) .{ }^{43}$ Dapagliflozin lowered the risk of end-stage renal disease or renal death $(\mathrm{p}=0.012)$ and cardiorenal and renal-specific composite endpoints were improved compared with placebo. ${ }^{44}$ Dapagliflozin has also been associated with much improved health-related quality of life (EQ-5D) scores that persisted for 102 weeks. ${ }^{41}$

\section{Dapagliflozin for the treatment of type 1 diabetes - The DEPICT studies}

Dapagliflozin has been evaluated in patients with T1D in the extensive, long-term phase III DEPICT clinical trial programme. 45,45,46

\section{Clinical benefits of dapagliflozin as adjunctive therapy in type 1 diabetes}

The DEPICT-1 and DEPICT-2 clinical trials had a similar design and differed only in different geographical patient populations. These trials evaluated the efficacy and safety of dapagliflozin (either $5 \mathrm{mg}$ or $10 \mathrm{mg}$ ) versus placebo as add-on to insulin in patients with T1D over 24 weeks followed by a 28-week extension period. ${ }^{4.5}$ These were double-blind, randomized, parallel-controlled, three-arm studies with patients ( $n=833$ and $n=813$ in DEPICT-1 and -2 , respectively) aged $18-75$ years with inadequately controlled T1D who had received insulin for at least 12 months. ${ }^{4,5}$ Patients treated with dapagliflozin (either $5 \mathrm{mg}$ or $10 \mathrm{mg}$ ) plus insulin showed significant improvement in adjusted mean change in $\mathrm{HbA} 1 \mathrm{C}$ from baseline by $-0.40 \%$ and $-0.43 \%$ compared with placebo after 24 weeks, respectively (pooled data from DEPICT-1 and -2) (Figure 1). ${ }^{4,5}$ After 52 weeks, the effect was numerically smaller (-0.26 and $-0.30 \%$ for dapagliflozin $5 \mathrm{mg}$ and $10 \mathrm{mg}$, respectively). ${ }^{47,48}$ The proportion of patients with $\mathrm{a} \geq 0.5 \%$ reduction in $\mathrm{HbA} 1 \mathrm{C}$ in the absence of severe hypoglycaemia, was significantly increased with dapagliflozin versus placebo at 24 weeks. $4,5,47$ This is important since the improvement in HbA1C did not increase the rate of hypoglycaemia in the DEPICT trials in contrast with increased hypoglycaemia with intensive insulin therapy as shown in the DCCT trial. ${ }^{49}$

In addition, time in range improved with both doses of dapagliflozin at 12 and 24 weeks (Figure 2) ${ }^{46}$ but without increasing time below range. Similarly, there was a reduction in adjusted mean percent change in total body weight after 24 and 52 weeks (DEPICT-1 and DEPICT-2 $p<0.0001$ for both doses versus placebo), and this effect was consistent over time. ${ }^{4,5}$ In DEPICT-1 total daily insulin dose was also significantly reduced by $8.8 \%$ 
Figure 1: DEPICT-1 and -2 primary endpoints, adjusted mean change in HbA1C at 24 weeks ${ }^{4,5}$

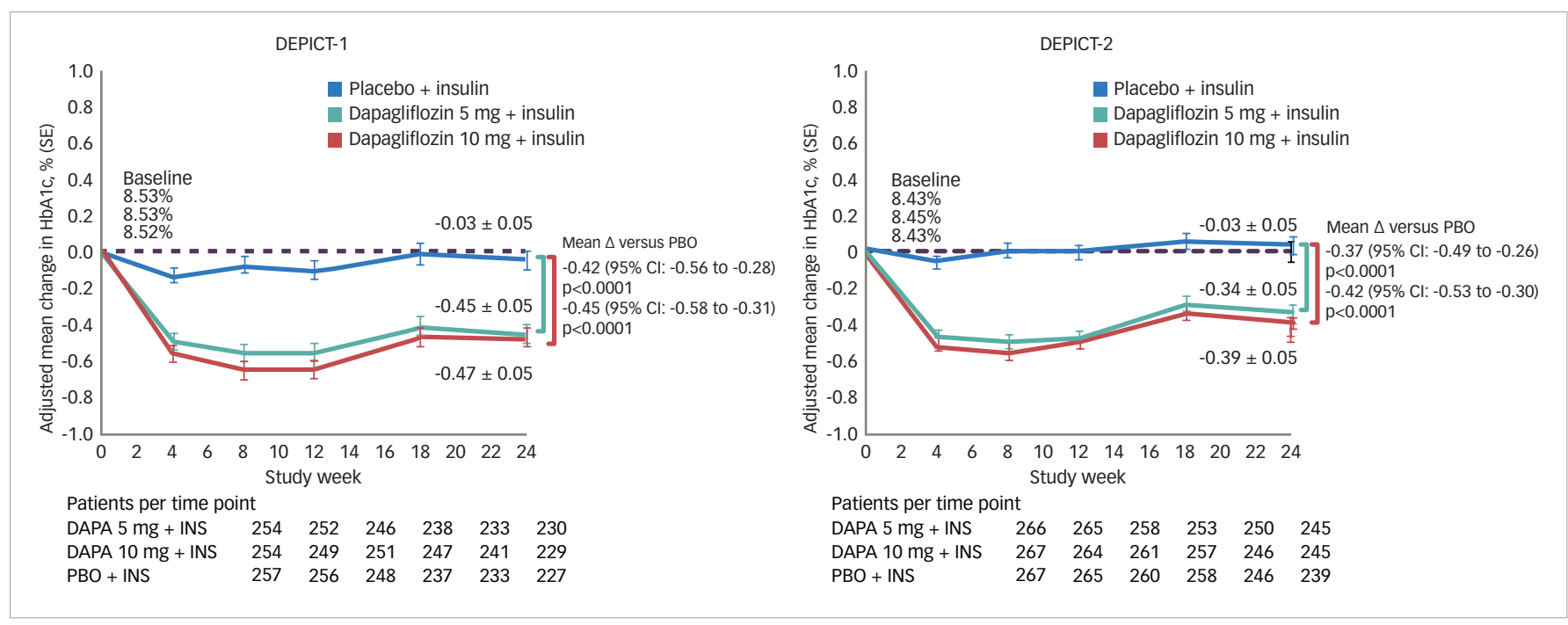

$\mathrm{Cl}=$ confidence interval; DAPA = dapagliflozin; DEPICT = Dapagliflozin Evaluation in Patients with Inadequately Controlled Type 1 diabetes; $\mathrm{HbA1C}=$ glycated haemoglobin; INS = insulin; $P B O=$ placebo; $S E=$ standard error.

Reproduced with permission from Dandona et al., $2017^{4}$ (Copyright Elsevier 2017) and Mathieu et al., 2018 (Efficacy and safety of dapagliflozin in patients with inadequately controlled type 1 diabetes (the DEPICT-2 study): 24-week results from a randomized controlled trial, American Diabetes Association, 2018. Copyright and all rights reserved. Material from this publication has been used with the permission of American Diabetes Association). ${ }^{5}$

Figure 2: Glucose time in range, hyperglycaemia and hypoglycaemia in the DEPICT-1 and -2 pooled continuous glucose monitoring 46

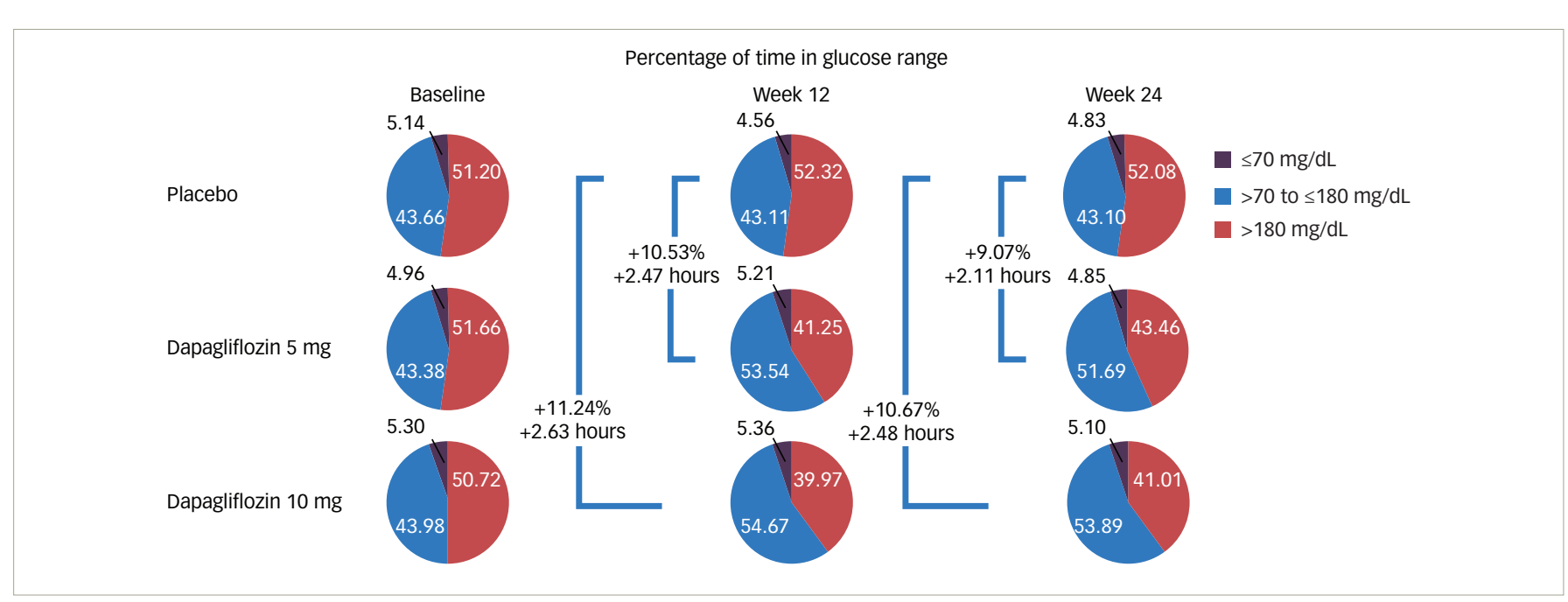

DEPICT = Dapagliflozin Evaluation in Patients with Inadequately Controlled Type 1 diabetes.

(95\% Cl: -12.6 to $-4.9 ; \mathrm{p}<0.0001)$ and $-13.2 \%$ (95\% Cl: -16.8 to $-9.4 ; \mathrm{p}<0.0001)$ for dapagliflozin $5 \mathrm{mg}$ and $10 \mathrm{mg}$ versus placebo, respectively, after 24 weeks. Mean difference in daily insulin dose per $\mathrm{kg}$ body weight at week 24 was $-5.5 \%$ (95\% Cl:-9.2 to - $1.6 ; p=0.0064$ ) and $-9.8 \%(95 \% \mathrm{Cl}:-13.4$ to $-6.1 ; p<0.0001)$ for dapagliflozin $5 \mathrm{mg}$ and $10 \mathrm{mg}$, respectively. Basal and bolus doses of insulin were decreased similarly to daily doses. Basal doses were $-11.6 \%$, (standard error [SE]: 1.3), -13.7\% (SE: 1.3) and -0.6\% (SE: 1.5) adjusted mean changes from baseline after 24 weeks for dapagliflozin $5 \mathrm{mg}, 10 \mathrm{mg}$ and placebo, respectively. Bolus doses were $-14.3 \%(2.1),-18.0 \%$ (2.1) and $-4.6 \%$ (2.4), respectively. ${ }^{450}$ Similar reductions in insulin doses were observed at 52 weeks. $^{51}$

In DEPICT-2 at week 24, the total daily insulin dose was reduced by both doses of dapagliflozin respectively $(-13.7,-7.7$ and $-14.0,-8.0 ; p<0.0001$ for both comparisons). These reductions were seen within the first 2 weeks and continued over the course of the study. Basal insulin adjusted mean changes were -11.2\% (SE: 1.5), -16.7\% (SE: 1.4) and 1.5\% (SE: 1.7) for $5 \mathrm{mg}, 10 \mathrm{mg}$ dapagliflozin and placebo, respectively. Results for bolus insulin were similar (-11.6\% [SE: 2.0], -8.3\% [SE: 2.1] and -2.6\% [SE: 2.2], respectively). ${ }^{5}$

\section{Continuous glucose monitoring from DEPICT-1 and DEPICT-2 pooled data set}

Twenty-four-hour mean interstitial glucose was reduced with dapagliflozin treatment but not with placebo treatment. Time in range was increased with both dapagliflozin doses at 12 and 24 weeks but in the placebo group it was slightly reduced. At week 24 , the adjusted mean (SE) change from baseline was $+6.5 \%(0.6),+8.1 \%(0.6)$ and $-2.6 \%$ (0.6) for dapagliflozin $5 \mathrm{mg}, 10 \mathrm{mg}$ and placebo, respectively. Mean time in target range expressed as the percentage of readings (standard deviation) was $51.7 \%$ (14.5\%) and 53.9\% (13.3\%) for dapagliflozin $5 \mathrm{mg}$ and $10 \mathrm{mg}$, respectively, and $43.1 \%$ (14.0\%) for placebo. At week 24 , 
the 24-hour glucose reading (hours) for time below range $(\leq 3.9 \mathrm{mmol} / \mathrm{L}$ [ $\leq 70 \mathrm{mg} / \mathrm{dL}]$ ) was $4.9 \%(5.0 \%), 5.1 \%(4.4 \%)$ and $4.8 \%(4.7 \%)$ for dapagliflozin $5 \mathrm{mg}, 10 \mathrm{mg}$ and placebo, respectively. Time above range $(>10 \mathrm{mmol} / \mathrm{L}$ [>180 mg/dL]) was $43.5 \%(10.4 \%), 41.0 \%(10.0 \%)$ and $52.1 \%(12.5 \%)$ for dapagliflozin $5 \mathrm{mg}, 10 \mathrm{mg}$ and placebo, respectively. Mean postprandial glucose values were also decreased in patients receiving dapagliflozin treatment. Regarding glycaemic variability, the adjusted mean change in mean amplitude of glucose excursion from baseline was reduced by both dapagliflozin doses at 12 and 24 weeks, being at week $24,-0.7 \mathrm{mmol} / \mathrm{L}(-12.5 \mathrm{mg} / \mathrm{dL})$, $-0.7 \mathrm{mmol} / \mathrm{L}(-13.1 \mathrm{mg} / \mathrm{dL})$ and $0.1 \mathrm{mmol} / \mathrm{L}(0.9 \mathrm{mg} / \mathrm{dL})$ for dapagliflozin $5 \mathrm{mg}, 10 \mathrm{mg}$ and placebo, respectively. ${ }^{9}$

\section{Safety of dapagliflozin in type 1 diabetes}

Results from DEPICT-1 indicated that dapagliflozin was generally well tolerated with six individuals (2.0\%) in the dapagliflozin $5 \mathrm{mg}$ group, eight (3.0\%) in the $10 \mathrm{mg}$ group and nine (3.0\%) in the placebo group discontinuing due to adverse events. One or more serious adverse events was recorded in 19 (7.0\%), 24 (8.0\%) and 15 (6.0\%) patients treated with dapagliflozin $5 \mathrm{mg}, 10 \mathrm{mg}$ and placebo, respectively. Severe hypoglycaemia was similar between groups occurring in 8.0\%, 6.0\% and $7.0 \%$ in patients treated with dapagliflozin $5 \mathrm{mg}, 10 \mathrm{mg}$ and placebo, respectively. ${ }^{4}$ After 24 weeks, genital infections occurred more frequently in dapagliflozin-treated patients compared with placebo and were more common in women; for dapagliflozin $5 \mathrm{mg}$, 25 of 158 women (16\%) versus 9 of 119 men (7.6\%); for $10 \mathrm{mg}$, 23 of 144 women (16.0\%) versus 10 of 152 men (6.6\%); and for placebo 7 of 128 women (5.5\%) versus none in 132 men. There were no reports of serious genital infection adverse events. ${ }^{4}$ Similarly, after 52 weeks, genital infections were reported more often in women than men $(21.5 \%$ versus $7.6 \%, 18.8 \%$ versus $8.6 \%$ and $6.3 \%$ versus $0 \%$, for women versus men in the dapagliflozin $5 \mathrm{mg}$, $10 \mathrm{mg}$ and placebo groups, respectively)..$^{50}$

In DEPICT-2, $\geq 1$ serious adverse event were seen in 18 (6.6\%) patients treated with dapagliflozin $5 \mathrm{mg}$, seven (2.6\%) patients treated with dapagliflozin $10 \mathrm{mg}$ and five $(1.8 \%)$ treated with placebo. Furthermore, 17 (6.3\%) in the dapagliflozin $5 \mathrm{mg}$ group, 12 (4.4\%) in the $10 \mathrm{mg}$ group and 11 (4.0\%) in the placebo group discontinued due to adverse events. In each treatment group a similar proportion of patients had hypoglycaemia $(82.3 \%, 85.6 \%$ and $86.0 \%$ in patients receiving $5 \mathrm{mg}, 10 \mathrm{mg}$ dapagliflozin and placebo, respectively) and severe hypoglycaemia $(6.3 \%, 8.5 \%$ and $7.7 \%$ in patients receiving $5 \mathrm{mg}, 10 \mathrm{mg}$ dapagliflozin and placebo, respectively). ${ }^{5}$ As in DEPICT-1, genital infections were more frequent in the treatment groups than placebo and were similar with both doses of dapagliflozin. They occurred in $15.7 \%$ versus $2.5 \%, 12.8 \%$ versus $1.7 \%$ and $3.3 \%$ versus none in the $5 \mathrm{mg}$, $10 \mathrm{mg}$ dapagliflozin and placebo groups, respectively, for women versus men after 24 weeks. No patients had serious genital infections. ${ }^{5}$

DKA is a major acute diabetes complication that is linked to premature death in patients with T1D. In the DEPICT-1 and -2 trials, an independent adjudication committee evaluated all potential DKA events. During the randomized period and follow-up, definite adjudicated DKA events occurred more frequently in those receiving $5 \mathrm{mg}$ or $10 \mathrm{mg}$ dapagliflozin than in the placebo groups (Table 1).,45 In DEPICT-1, an interactive voice response system error resulted in a randomization error affecting the first 55 patients and these were excluded from the efficacy analysis set, but included in the safety analysis set, 14 of these patients were non-randomly allocated to the dapagliflozin $5 \mathrm{mg}$ group. However, in DEPICT-1 and -2, the subgroup of patients with a BMI of $\geq 27 \mathrm{~kg} / \mathrm{m}^{2}$ had fewer definite DKA events than
Table 1: Frequency of diabetic ketoacidosis in clinical trials of SGLT2 inhibitors ${ }^{4-7}$

\begin{tabular}{|c|c|c|c|c|}
\hline Trial & Drug & $\mathrm{N}$ & $\begin{array}{l}\text { DKA } \\
\text { frequency } \\
(\%)\end{array}$ & $\begin{array}{l}\text { Dose } \\
\text { (mg) }\end{array}$ \\
\hline \multirow[t]{3}{*}{ DEPICT-1 } & \multirow[t]{3}{*}{ Dapagliflozin } & \multirow[t]{3}{*}{833} & 1.0 & 5 \\
\hline & & & 2.0 & 10 \\
\hline & & & 1.0 & Placebo \\
\hline \multirow[t]{3}{*}{ DEPICT-2 } & \multirow[t]{3}{*}{ Dapagliflozin } & \multirow[t]{3}{*}{813} & 2.6 & 5 \\
\hline & & & 2.2 & 10 \\
\hline & & & 0.0 & Placebo \\
\hline \multirow{3}{*}{$\begin{array}{l}\text { DEPICT-1, -2 pooled } \\
\text { total population }\end{array}$} & \multirow[t]{3}{*}{ Dapagliflozin } & \multirow[t]{3}{*}{1,646} & 4.0 & 5 \\
\hline & & & 1.1 & 10 \\
\hline & & & 1.1 & Placebo \\
\hline \multirow{2}{*}{$\begin{array}{l}\text { DEPICT-1, }-2 \text { pooled, } \\
\text { BMI } \geq 27 \mathrm{~kg} / \mathrm{m}^{2}\end{array}$} & \multirow[t]{2}{*}{ Dapagliflozin } & \multirow[t]{2}{*}{561} & 1.8 & 5 \\
\hline & & & 1.0 & Placebo \\
\hline \multirow[t]{2}{*}{ inTandem3 } & \multirow[t]{2}{*}{ Sotagliflozin } & \multirow[t]{2}{*}{1,402} & 3.0 & 400 \\
\hline & & & 0.6 & Placebo \\
\hline \multirow[t]{4}{*}{ EASE-1,2,3 } & \multirow[t]{4}{*}{ Empagliflozin } & \multirow[t]{4}{*}{1,707} & 4.3 & 10 \\
\hline & & & 3.3 & 25 \\
\hline & & & 0.8 & 2.5 \\
\hline & & & 1.2 & Placebo \\
\hline
\end{tabular}

$B M I=$ body mass index; DEPICT = Dapagliflozin Evaluation in Patients with Inadequately Controlled Type 1 diabetes; DKA = diabetic ketoacidosis; inTandem = as adjunct to insulin; EASE = Empagliflozin as Adjunctive to insulin therapy; SGLT2 = sodium-glucose co-transporter-2.

the overall population and the benefit-risk profile is enhanced in these patients. ${ }^{39}$ This is discussed further in the next section.

\section{Benefit-risk analysis in the subgroup of patients with body mass index $\geq 27 \mathrm{~kg} / \mathrm{m}^{2}$ in DEPICT-1 and -2} In DEPICT-1 and -2, after 52 weeks of treatment in the subgroup of patients with a $\mathrm{BMI} \geq 27 \mathrm{~kg} / \mathrm{m}^{2}$, efficacy was comparable to that in the overall population..$^{39}$ In the total population, the proportions of patients who experienced definite DKA events during the study were $4.0 \%$ and $1.1 \%$ in patients treated with dapagliflozin $5 \mathrm{mg}$ or placebo, respectively, while the incidence of DKA was 4.6 and 1.3 per 100 patient years (Figure 3). ${ }^{39,40}$ However, in patients with a BMI $\geq 27 \mathrm{~kg} / \mathrm{m}^{2}$, the proportion with DKA was $1.8 \%$ and $1.0 \%$ and incidence rates were 1.9 and 1.2 per 100 patient years in those treated with $5 \mathrm{mg}$ dapagliflozin or placebo, respectively. In addition, patients with a $\mathrm{BMl} \geq 27 \mathrm{~kg} / \mathrm{m}^{2}$ treated with dapagliflozin experienced more time in range compared with those treated with placebo (Figure 4$)^{40}$ and a higher proportion achieved a reduction in $\mathrm{HbA} 1 \mathrm{C} \geq 0.5 \%$ without experiencing hypoglycaemia (Figure 5). ${ }^{40}$ Thus, this post-hoc analysis suggests that the benefit-risk is enhanced in patients with a BMl $\geq 27 \mathrm{~kg} / \mathrm{m}^{2}$ treated with dapagliflozin $5 \mathrm{mg}$ compared with the complete DEPICT population. ${ }^{39}$

\section{Background risk of diabetic ketoacidosis in type 1 diabetes}

The frequency of DKA was assessed in the USA T1D Exchange Clinical Registry in people aged 26-93 years with T1D, and showed that $4.8 \%$ of 6,796 participants experienced one or more DKA events in 12 months. ${ }^{16}$ Furthermore, information from 25,833 adults and children recorded in this registry indicated that DKA occurred in $8.0 \%$ of patients with diabetes in a year..$^{13} \mathrm{~A}$ recent study from the Diabetes Prospective Follow-up Registry, including 46,966 patients (average age 38.5 years [median 21.2]), 
Figure 3: Definite diabetic ketoacidosis reduced in the subgroup of patients with body mass index $\geq 27 \mathrm{~kg} / \mathrm{m}^{2}$ at week 52 DEPICT-1 and $-2^{39}$

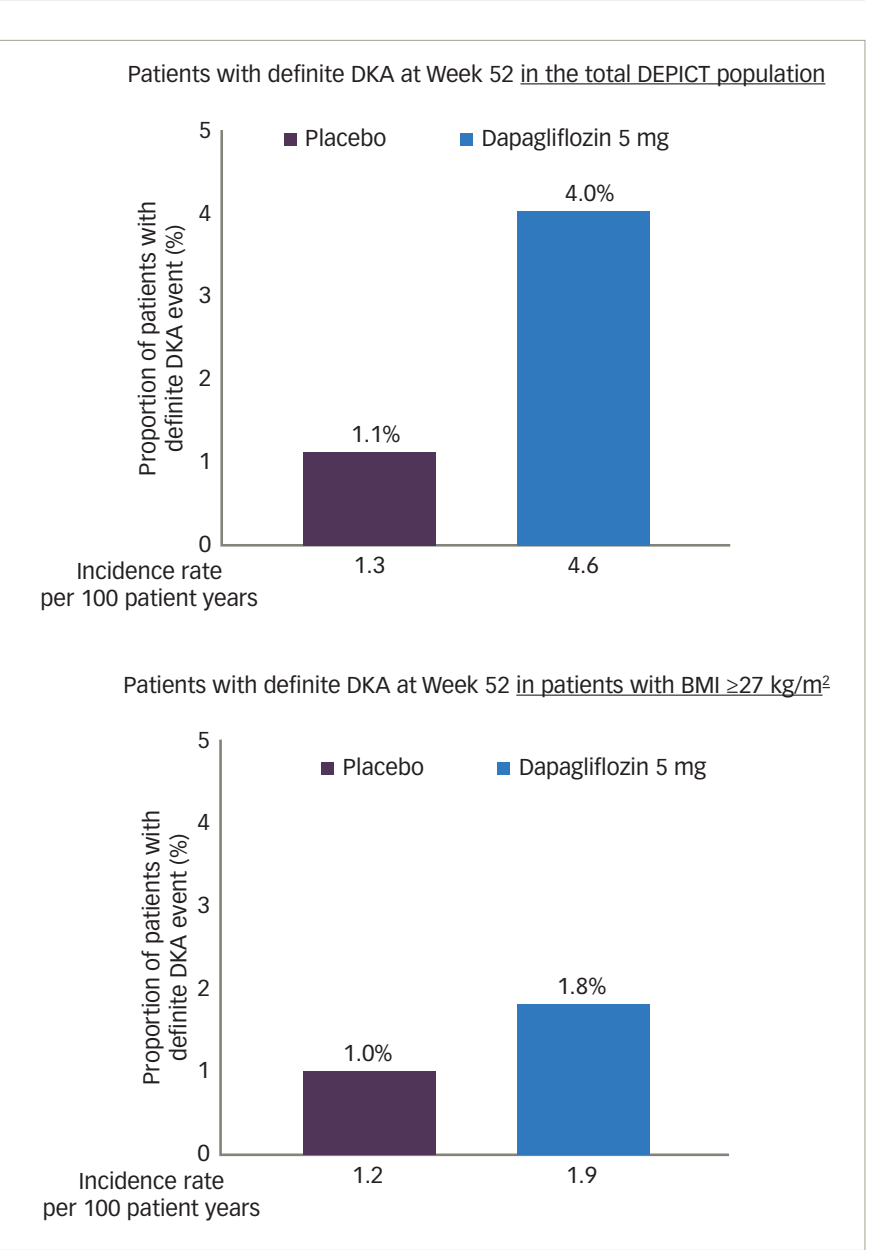

$B M I=$ body mass index; DEPICT = Dapagliflozin Evaluation in Patients with Inadequately Controlled Type 1 diabetes; DKA = diabetic ketoacidosis. Reproduced with permission from Mathieu C, et al., 2019. ${ }^{40}$ Copyright Elsevier 2019.

Figure 4: Subgroup analysis of patients with body mass index $\geq 27 \mathrm{~kg} / \mathrm{m}^{2}$

DAPA $5 \mathrm{mg}+$ insulin

Difference versus placebo + insulin at week 24

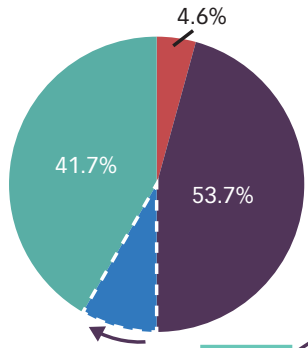

Time in range $=+9.69 \%$

Time in hypoglycaemia: $+0.01 \%$

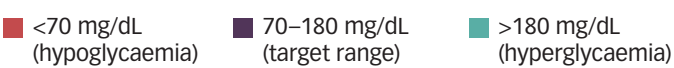

More time in glycaemic range without increasing time in hypoglycaemia at 24 weeks DEPICT-1 and $-2{ }^{39}$

Mixed model: change from baseline = baseline treatment study week stratum week, treatment week, baseline subgroup treatment, subgroup week, subgroup and treatment, week, subgroup (stratum includes one term for each combination of all stratification factors).

DAPA = dapagliflozin; DEPICT $=$ Dapagliflozin Evaluation in Patients with Inadequately Controlled Type 1 diabetes.

Reproduced with permission from Mathieu et al., 2019. ${ }^{40}$ Copyright Elsevier 2019.
Figure 5: Subgroup analysis of patients with body mass index $\geq 27 \mathrm{~kg} / \mathrm{m}^{2}$

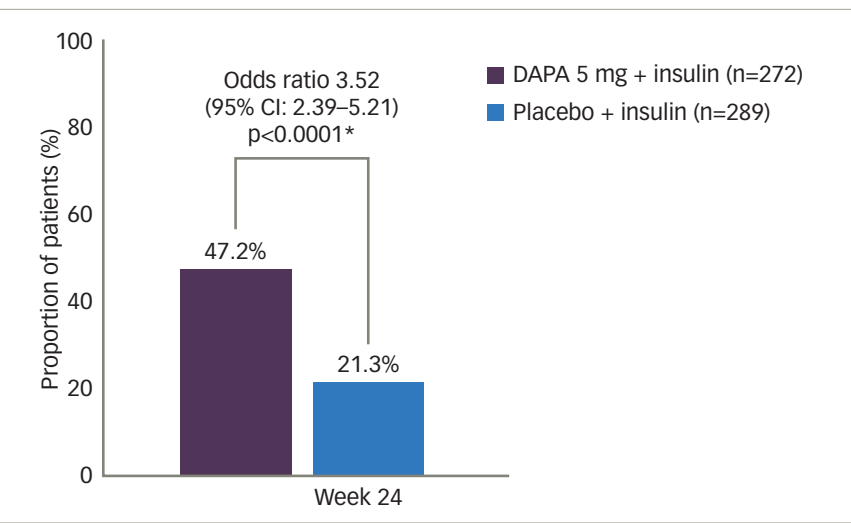

A higher proportion of patients achieved a reduction in $\mathrm{HBA} 1 \mathrm{C} \geq 0.5 \%$ without experiencing severe hypoglycaemia at 24 weeks DEPICT-1 and $-2 .{ }^{39}$ *Nominal p-value: logistic regression adjusted for baseline HbA1C study and randomization strata.

$\mathrm{Cl}=$ confidence interval; DAPA = dapagliflozin; DEPICT = Dapagliflozin Evaluation in Patients with Inadequately Controlled Type 1 diabetes; HDA1C = glycated haemoglobin. Reproduced with permission from Mathieu et al., 2019. ${ }^{40}$ Copyright Elsevier 2019.

found, on average, 2.5 DKA-related hospital admissions per 100 patient years (95\% Cl: 2.1-3.0). The rate was highest in patients aged 18-30 years (4.03/100 patient years) and gradually declined with increasing age. ${ }^{52}$ No meaningful improvements in the frequency of DKA have been achieved over the last decade despite improvement in insulin therapy. Insulin is the mainstay of treatment for T1D. If there is an insulin deficit, excess ketone body formation ensues and DKA may occur. There is thus an urgent need for in-depth education to prevent DKA.

\section{Potential cardiovascular and renal benefits of dapagliflozin in type 1 diabetes}

SGLT2 inhibitors have a beneficial effect on MACE in patients with T2D. ${ }^{53}$ Overall, a meta-analysis of EMPA-REG OUTCOME, CANVAS and DECLARE trials showed an $11.0 \%$ reduction in MACE $(p=0.0014){ }^{54}$ It is known that patients with T1D have an increased risk of hospitalization for heart failure. ${ }^{54}$ SGLT2 inhibitors reduce the risk for hospitalization for heart failure, cardiovascular mortality and progression to chronic kidney disease in patients with T2D.5. T1D is also associated with microvascular complications, but the onset and progression of retinopathy is delayed in patients who have better glycaemic control. ${ }^{28}$

\section{Effect of adding dapagliflozin on urinary albumin-to-creatinine ratio from DEPICT-1 and DEPICT-2 pooled data set over 52 weeks}

Patients with T1D also have a high risk of end stage renal disease despite the use of renoprotective treatment. ${ }^{56}$ In patients with albuminuria at baseline ( $\geq 30 \mathrm{mg} / \mathrm{g}$ ), adjusted mean change in $\mathrm{HbA} 1 \mathrm{C}$ levels, systolic blood pressure, urinary albumin-to-creatinine ratio and estimated glomerular filtration rate were improved in those treated with dapagliflozin compared with placebo. The difference versus placebo in urinary albumin-to-creatinine ratio was -13.3 and $-31.1 \mathrm{mg} / \mathrm{g}$ with dapagliflozin $5 \mathrm{mg}$ and $10 \mathrm{mg}$, respectively. ${ }^{55}$

\section{Dapagliflozin - patient selection and education}

Reductions in $\mathrm{HbA} 1 \mathrm{C}$, weight and blood pressure are similar among the different SGLT2 inhibitors evaluated in patients with T1D (dapagliflozin, empagliflozin and sotagliflozin [a dual SGLT1 and SGLT2 inhibitor]). ${ }^{57}$ All studies reported that the therapies can slightly increase the risk of DKA in clinical trial settings (Table 1). In the inTandem3 trial of 
Figure 6: Ketone monitoring levels and action required 56

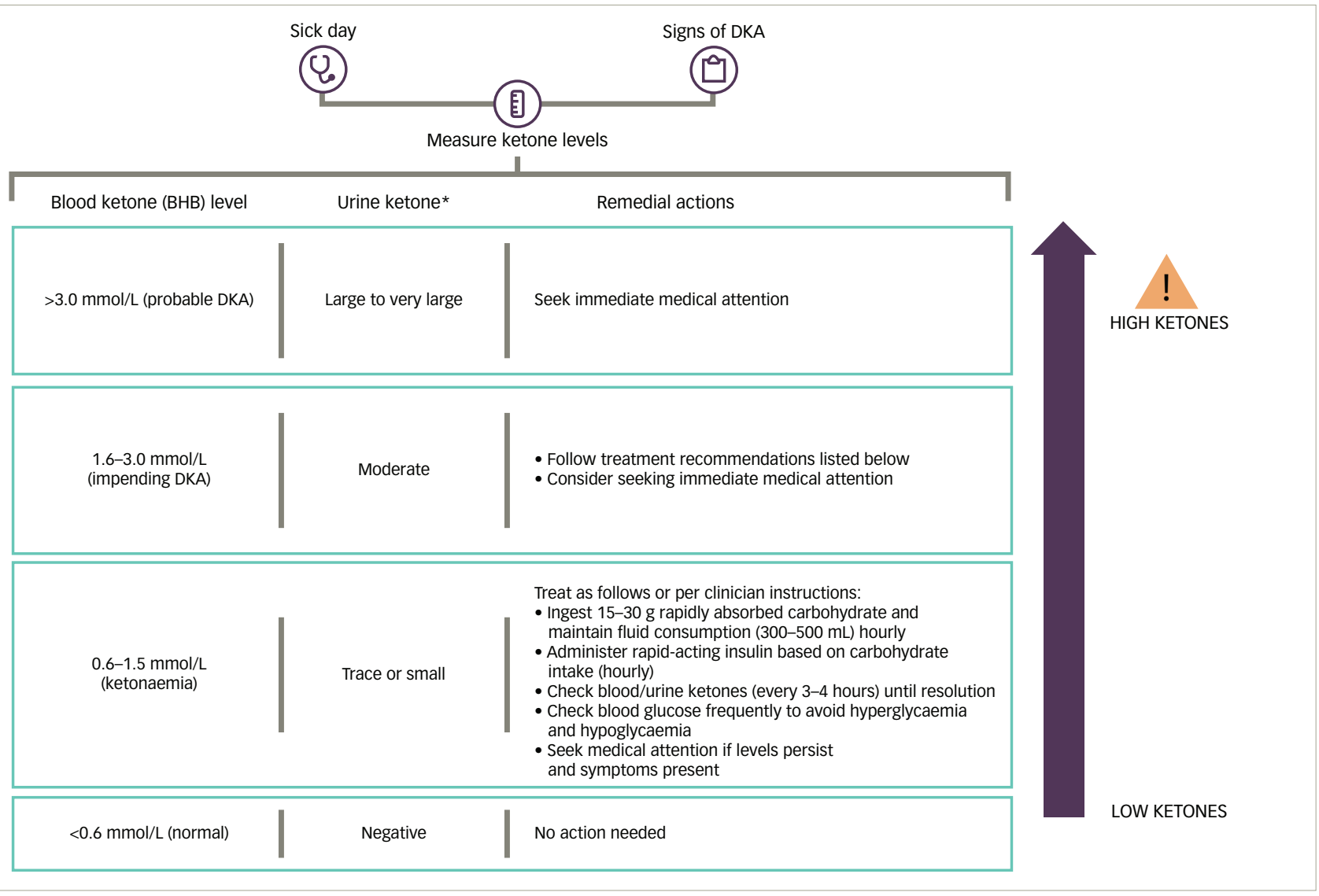

It is important to have blood ketone analysis available for the patients on SGLT2 inhibitors when treating type 1 diabetes with adjunctive SGLT2 inhibitors to multiple daily insulin injections or continuous subcutaneous insulin infusions.

*Urine ketone concentrations are dependent on hydration and other factors; these values do not closely correlate with blood BHB levels. $B H B=\beta$-hydroxybutyrate; $D K A=$ diabetic ketoacidosis; $S G L T 2$ = sodium-g/ucose co-transporter-2.

Reproduced with permission from Danne et al., 2019. ${ }^{56}$ Copyright Elsevier 2019.

sotagliflozin, patients had no episodes of severe hypoglycaemia, and the rate of documented hypoglycaemia was similar in the sotagliflozin and placebo groups. DKA occurred more often in the sotagliflozin group. 7.8 Results from the inTandem 1 and -2 were similar. The EASE-1,2,3 trials evaluated empagliflozin as adjunctive to insulin therapy in patients with T1D and showed reductions in $\mathrm{HBA} 1 \mathrm{C}$, weight, total daily insulin dose and systolic blood pressure. Adjudicated DKA was more frequently observed with empagliflozin, at $4.3 \%, 3.3 \%$, $0.8 \%$ and $1.2 \%$ with $10 \mathrm{mg}, 25 \mathrm{mg}, 2.5 \mathrm{mg}$ and placebo, respectively, indicating that lower doses are associated with a decreased risk of DKA. ${ }^{6}$

A consensus report published in 2019 gave recommendations for improving the safety of SGLT2 inhibitors in people with T1D, such as reducing with caution initial insulin doses, not stopping insulin in any patient, measurement of blood or urine ketones when necessary, and improvement in patient and clinician education with respect to preventing ketosis episodes. ${ }^{56}$ The European Medicine Agency reviewed the consensus statement and executed an expert hearing during the approval process. Both were brought together and combined into the final summary of product characteristics for dapagliflozin in T1D with the recommended dose being $5 \mathrm{mg}$ per day..$^{58}$

According to the European label, dapagliflozin $5 \mathrm{mg}$ should only be administered for the treatment of T1D to adult patients whose BMI is $\geq 27 \mathrm{~kg} / \mathrm{m}^{2}$ when there is inadequate glycaemic control despite optimal insulin therapy. Before starting treatment, the benefits should be weighed against the risks, particularly of DKA, in each patient. A risk mitigation plan has been developed for DKA. ${ }^{59}$ Before initiating dapagliflozin, the doctor should assess risk factors that could predispose the patient to DKA and educate them to recognize the characteristic signs of ketosis and know how and when to monitor blood ketones. The patient must be able and willing to monitor ketone levels, have access to both testing materials and a clinician when raised levels are identified (Figure 6). ${ }^{56}$

When using SGLT2 inhibitors, DKA may be difficult to diagnose as people can have normal blood glucose levels or merely mild hyperglycaemia. Insulin therapy should be optimized during dapagliflozin treatment to prevent DKA, and glucose monitoring must be supplemented by ketone monitoring when necessary. ${ }^{58}$ An independent DKA education programme has recently been developed to educate patients with T1D treated with and without both insulin pumps and SGLT2 inhibitor therapy. It is currently available in English and German but will be translated into other languages. ${ }^{60}$

\section{Discussion}

T1D is a lifelong condition that causes an 11-13 year reduction in life expectancy due to acute metabolic complications in younger individuals and cardiovascular disease in older individuals. ${ }^{31}$ SGLT2 inhibitors have been shown to be effective in both T2D and T1D. SGLT inhibitors in people with T1D improve glycaemic control and weight while reducing insulin doses without increasing the risk of hypoglycaemia. ${ }^{61}$ 
Several serious unmet needs remain in the treatment of T1D, notably insufficient glycaemic control, increasing weight and higher risk of hypoglycaemic events. Dapagliflozin treatment addresses these unmet needs. Glycaemic variability is often associated with hypoglycaemia, especially severe hypoglycaemia, which may have detrimental long-term consequences. The reduction in glycaemic variability with dapagliflozin may diminish the risk of hypoglycaemia and may allow a more predictable insulin dosing. There is a known background risk of DKA in people with T1D. Despite SGLT inhibitors slightly increasing this risk of DKA, with appropriate diabetes education this risk is manageable.

Findings from the DCCT and EDIC studies indicate that intensive insulin treatment reduced the number of any cardiovascular events compared with conventional treatment. More recent studies have also shown that the risk of all-cause death and cardiovascular death is strongly linked to high HbA1c levels. ${ }^{62}$ The follow-up study to the DCCT and EDIC study, showed that the long-term benefit of intensive insulin treatment on reducing cardiovascular events was not seen in those who gained the most weight. ${ }^{24}$ The DCCT also demonstrated the importance of glycaemic control on preventing microvascular outcomes. ${ }^{63}$ In particular, increasing estimated time in range (70-180 $\mathrm{mg} / \mathrm{dL})$ has a beneficial effect on preventing microvascular complications. ${ }^{30}$

Dapagliflozin treatment has been assessed in a number of clinical trials in patients with T2D as monotherapy and in combination with insulin and other anti-diabetic therapies. ${ }^{4,5}$ Clinical trials evaluating dapagliflozin as adjunct to insulin therapy in patients with T1D have demonstrated that adjusted mean change in HbA1C is improved, body weight is decreased and daily insulin dose is reduced. ${ }^{64}$ Similar results were obtained with sotagliflozin in the inTandem trials and with empagliflozin in the EASE trial. ${ }^{6.8}$ As weight gain is a major problem in patients with T1D and often occurs in patients on intensive insulin treatment, the reduction in body weight with dapagliflozin is remarkable.

Dapagliflozin $5 \mathrm{mg}$ is now indicated for the treatment of T1D in the EU. Nevertheless, it should be noted that in the EU, dapagliflozin is only approved for patients with T1D with a BMI $\geq 27 \mathrm{~kg} / \mathrm{m}^{2}$, in whom insulin gives inadequate glycaemic control. ${ }^{36} \mathrm{~A}$ benefit-risk analysis in the subgroup of patients with a BMI $\geq 27 \mathrm{~kg} / \mathrm{m}^{2}$ in the large-scale DEPICT-1 and -2 studies showed that glycaemia and body weight decreased from baseline with dapagliflozin $5 \mathrm{mg}$ compared with patients receiving placebo similarly to the total study population while reducing the risk for
DKA by more than half. This population of overweight patients with T1D is increasing all over the world. ${ }^{1.65}$ Overweight and obese patients and those with latent autoimmune diabetes in adults should be considered for treatment with SGLT2 inhibitors as adjunctive therapy to insulin because of the low risk of DKA, although to date, no clinical trials have been initiated in latent autoimmune diabetes. ${ }^{66,67}$

Patients must be educated with respect to DKA and be able to recognize the symptoms and the situations that may increase the risk of this event. Dapagliflozin is generally well tolerated with ketone-related side effects being the most notable adverse events, but these are dose dependent and occur at a low frequency. However, treating patients with T1D with dapagliflozin $5 \mathrm{mg}$ in a real-world setting will require close and permanent monitoring regarding adherence and compliance with the safety measures to avoid the occurrence of DKA. Long-term and prospectively collected data from greater numbers of patients in daily clinical practice will be needed to identify patients who will have the least risk for DKA but will have the maximum benefit. ${ }^{34}$

In DEPICT-1 no increase in severe hypoglycaemia or in the overall incidence of hypoglycaemia was observed. In addition, urinary albumin-to-creatinine ratio was reduced in patients with albuminuria at baseline in the DEPICT clinical trials of dapagliflozin treatment, which suggests a putative renoprotective effect. ${ }^{55}$

Finally, there is a potential for transferability of outcomes of SGLT2 inhibition between T2D and T1D. The risk-benefit assessment to initiate an SGLT2 inhibitor in patients with T1D should include the broader knowledge of the positive effect beyond glucose control of reducing the risk of major cardiovascular and renal outcomes. ${ }^{68}$

In summary, dapagliflozin addresses some of the unmet needs associated with T1D by improving glycaemic control with weight loss and without increasing hypoglycaemia, by reducing glycaemic variability. Genital infections were more frequent as seen in patients with T2D. DKA events were slightly increased but may be prevented by an adequate selection of patients and an appropriate mitigation education programme. Overall, the data collected from clinical trials, as well as considerable experience in treating patients with T2D with or without concomitant insulin treatment, indicate that the SGLT2 inhibitors, in particular dapagliflozin, have a valuable current and future role in the successful management of T1D. $\square$
1. International Diabetes Federation. IDF Diabetes Atlas 9th edition 2019, 2019. Available at: www.diabetesatlas.org/en/ resources/ (accessed 11 August 2020)

2. Patterson CC, Harjutsalo V, Rosenbauer J, et al. Trends and cyclical variation in the incidence of childhood type 1 diabetes cyclical variation in the incide in 26 European centres in the 25 year period 1989-2013: a multicentre prospe

3. Dahlquist $\mathrm{GG}$, Nyström $\mathrm{L}$, Patterson $\mathrm{CC}$, et al. Incidence of type 1 diabetes in Sweden among individuals aged 0-34 years, 1983-2007. Diabetes Care. 2011;34:1754-9.

4. Dandona P, Mathieu C, Phillip M, et al. Efficacy and safety of dapaglifozin in patents with inadequately controlled type 1 diabetes (DEPICT-1): 24 week results from a multicentre, double-blind, phase 3, randomised controlled trial. Lancet Diabetes Endocrinol. 2017:5:864-76.

5. Mathieu C, Dandona P, Gillard P, et al. Efficacy and safety of dapagliflozin in patients with inadequately controlled type 1 diabetes (the DEPICT-2 study): 24-week results from a randomized controlled trial. Diabetes Care 2018:41:1938-46.

6. Rosenstock J Marquard 1 feffel $1 M$, et al Empagliflozin as adjunctive to insulin therapy in type 1 diabetes: the EASE trials.

7. Garg SK, Henry RR, Banks P, et al. Effects of sotagliflozin added to insulin in patients with type 1 diabetes. $N$ Eng/ I Med. 2017;377:2337-48.

8. MCCrimmon RJ, Henry RR. SGLT inhibitor adjunct therapy in type 1 diabetes. Diabetologia. 2018;61:2126-33.
9. Mathieu C, Dandona P, Phillip M, et al. Glucose variables in type 1 diabetes studies with dapagliflozin: pooled analysis of continuous glucose monitoring data from DEPICT-1 and -2 . Diabetes Care. 2019;42:1081-7.

10. Conway B, Miller RG, Costacou T, et al. Temporal patterns in overweight and obesity in type 1 diabetes. Diabet Med. 2010:27:398-404.

11. Purnell JQ, Hokanson JE, Marcovina SM, et al. Effect of excessive weight gain with intensive therapy of type 1 diabetes on lipid levels and blood pressure: results from the DCCT. AMA. 1998;280:140-6.

12. McKnight JA, Wild SH, Lamb MJ, et al. Glycaemic control of type 1 diabetes in clinical practice early in the 21st century: an international comparison. Diabet Med. 2015;32:1036-50.

13. Beck RW, Tamborlane WW, Bergenstal RM, et al. The T1D Exchange Clinical Registry. I Clin Endocrinol Metab. 2012;97:4383-9.

14. Nationella Diabetesregistret. Swedish national diabetes register: Annual report 2013, 1-44, 2013. Available at: www.ndr.nu/pdfs/ Annual_Report_NDR_2013.pdf (accessed 11 August 2020).

15. Renard E, Pozzilli P, Wilmot E, et al. Suboptimal glycaemic control globally in all age groups of adults with type 1 diabetes: results of a multinational, observational study (SAGE). diabetes: results of a multinational, observational study (SAGE).
Presented at: European Association for the Study of Diabetes Presented at. European Association for the Study Annual Meeting, Barcelona, 2019. Available at:
www.easd.org/virtualmeeting/home.html\#!resources/ www.easd.org/virtualmeeting/home.html\#!resources/
suboptimal-glycaemic-control-globally-in-all-age-groupssuboptimal-glycaemic-control-globally-in-all-age-groups-
of-adults-with-type-1-diabetes-results-of-a-multinational- observational-study-sage-621e4c26-a6e1-498b-9dc86194cb7e8398 (accessed 11 August 2020).

16. Weinstock RS, Xing D, Maahs DM, et al. Severe hypoglycemia and diabetic ketoacidosis in adults with type 1 diabetes: results from the T1D exchange clinic registry. I Clin Endocrinol Metab. 2013:98:3411-19.

17. Gruden G, Barutta F, Chaturvedi N, et al. Severe hypoglycemia and cardiovascular disease incidence in type 1 diabetes. Diabetes Care 2012:35:1598-604

18. DeVries JH. glucose variability: where it is important and how to measure it. Diabetes. 2013;62:1405-8.

19. Ceriello A, Monnier L, Owens D. Glycaemic variability in diabetes: clinical and therapeutic implications. Lancet Diabetes Endocrinol. 2019;7:221-30.

20. Gerbaud E, Darier R, Montaudon M, et al. Glycemic variability is a powerful independent predictive factor of midterm majo adverse cardiac events in patients with diabetes with acute coronary syndrome. Diabetes Care. 2019;42:674-81.

21. Virk SA, Donaghue $\mathrm{KC}, \mathrm{Cho}$ YH, et al. Association between HDA1C variability and risk of microvascular complications in adolescents with type 1 diabetes. I Clin Endocrinol Metab. 2016;101:3257-63

22. Monnier L, Wojtusciszyn A, Molinari N et al. Respective contributions of glycemic variability and mean daily glucose as predictors of hypoglycemia in type 1 diabetes: are they equivalent? Diabetes Care. 2020;43:821-7.

23. Battelino T, Danne T, Bergenstal RM, et al. Clinical target for continuous glucose monitoring data interpretation: 
recommendations from the international consensus on time in range. Diabetes Care. 2019;42:1593-603.

24. Nathan DM, Cleary PA, Backlund J-YC, et al; DCCT/EDIC Study Research Group. Intensive diabetes treatment and cardiovascular disease in patients with type 1 diabetes. cardiovascular disease in patients

25. Purnell JQ, Braffett BH, Zinman B, et al. Impact of excessive weight gain on cardiovascular outcomes in type 1 diabetes: results from the Diabetes Control and Complications Trial/ Epidemiology of Diabetes Interventions and Complications (DCCT/EDIC) Study. Diabetes Care. 2017;40:1756-62

26. de Ferranti SD, de Boer IH, Fonseca V, et al. Type 1 diabetes mellitus and cardiovascular disease: a scientific statement from the American Heart Association and American Diabetes Association. Diabetes Care. 2014;37:2843-63.

27. Lithovius R, Toppila I, Harjutsalo V, et al. Data-driven metabolic subtypes predict future adverse events in individuals with type 1 diabetes. Diabetologia. 2017:60:1234-43.

28. Rawshani A, Sattar N, Franzén S, et al. Excess mortality and cardiovascular disease in young adults with type 1 diabetes in relation to age of onset: a nationwide, registry-based cohort study. Lancet. 2018:392:477-86.

29. de Boer IH, Rue TC, Cleary PA, et al. Long-term renal outcomes of patients with type 1 diabetes and microalbuminuria: an analysis of the DCCT/EDIC cohort. Arch Intern Med. 2011;171:412-20.

30. Aliello LP. Diabetic retinopathy and other ocular findings in the Diabetes Control and Complications Trial/Epidemiology of Diabetes Interventions and Complications Study. Diabetes Care. 2014;37:17-23.

31. Beck RW, Bergenstal RM, Riddlesworth TD, et al. Validation of time in range as an outcome measure for diabetes clinica trials. Diabetes Care. 2019:42:400-5

32. Livingstone SJ, Levin D, Looker HC, et al. Estimated life expectancy in a Scottish cohort with type 1 diabetes, 2008-2010. JAMA. 2015:313:37-44.

33. Song P, Onishi A, Koepsell $H$, et al. Sodium glucose cotransporter SGIT1 as a therapeutic target in diabetes mellitus. Expert Opin Ther Targets. 2016;20:1109-25.

34. Thynne T, Doogue M. Sodium-glucose co-transporter inhibitors Mechanisms of action. Aust Prescr. 2014;37:14-16.

35. Riddle MC, Cefalu WT. SGLT inhibitors for type 1 diabetes: an obvious choice or too good to be true? Diabetes Care 2018;41:2444-7.

36. AJMC. Sotagliflozin approved in EU for adults with type 1 diabetes, 2019. Available at: www.ajmc.com/view/sotagliflozinapproved-in-eu-for-adults-with-type-1-diabetes (accessed 11 August 2020).

37. AstraZeneca. Forxiga approved in Europe for type-1 diabetes, 2019. Available at: www.astrazeneca.com/media-centre/ press-releases/2019/forxiga-approved-in-europe-for-type-1 press-releases/2019/forxiga-approved-in-europe-for-typ
diabetes22032019.html (accessed 11 August 2020).

38. Lupsa BC, Inzucchi SE. Use of SGLT2 inhibitors in type 2 diabetes: weighing the risks and benefits. Diabetologia. 2018;61:2118-25.
39. Fattah $\mathrm{H}$, Vallon $\mathrm{V}$. The potential role of SGLT2 inhibitors the treatment of type 1 diabetes mellitus. Drugs. 2018;78:717-26.

40. Mathieu C, Dandona P, Phillip M, et al. Analysis of benefit/ risk in the subgroup of patients with BMI of $\geq 27 \mathrm{~kg} / \mathrm{m}^{2}$ in the risk in the subgroup of patients with BMl of $\geq 27 \mathrm{Kg} / \mathrm{m}^{2}$
dapaglifozin DEPICT- 1 and -2 trials in type 1 diabetes. J Endocr SOC. 2019;3(Suppl.1):SAT-LBO25.

41. McGurnaghan SJ, Brierlay L, Caparrotta, et al. The effect of dapagliflozin on glycaemic control and other cardiovascular disease risk factors in type 2 diabetes mellitus: a real-world observational study. Diabetologia. 2019;62:621-32.

42. Saeed MA, Narendran P. Dapagliflozin for the treatment of type 2 diabetes: a review of the literature. Drug Des Devel Ther. 2014;8:2493-505

43. Wiviott SD, Raz I, Bonaca MP, at al. Dapagliflozin and cardiovascular outcomes in type 2 diabetes. N Eng/ I Med. 2019;380:347-57

44. Mosenzon O, Wiviott SD, Cahn A, et al. Effects of dapagliflozin on development and progression of kidney disease in patients with type2 diabetes: an analysis from the DECLARE-TIMI 58 randomised trial. Lancet Diabetes Endocrinol. 2019:7:606-17.

45. US National Library of Medicine. Effects of single doses of liraglutide and dapagliflozin on hyperglycemia and ketogenesis in type 1 diabetes (1974), 2017. Available at: https://clinicaltria s. gov/ct2/show/NCT02777073 (accessed 11 August 2020)

46. US National Library of Medicine. The safety and efficacy of dapagliflozin therapy in combination with insulin in Japanese subjects with T1DM, 2019. Available at: https://clinicaltrials.gov/ ct2/show/NCT02582814 (accessed 11 August 2020).

47. Mathieu C, Rudofsky G, Phillip M, et al. Long-term efficacy and safety of dapagliflozin in patients with inadequately controlled type 1 diabetes (the DEPICT-2 study): 52-week results from a randomized controlled trial. Diabetes Obes Metab. 2020;22:1516-26.

48. Dandona P, Mathieu C, Phillip M, et al. Dapagliflozin (DAPA) in type 1 diabetes (T1D): pooled outcomes from DEPICT-1 and -2. Diabetes. 2019;68(Suppl. 1):1231-P.

49. The Diabetes Control and Complications Trial Research Group. The effect of intensive treatment of diabetes on the development and progression of long-term complications in insulin-dependent diabetes mellitus. N Eng/ J Med. 1993;329:977-86

50. Paik J, Blair HA. Dapagliflozin: a review in type 1 diabetes. Drugs. 2019;79:1877-84

51. Dandona P, Mathieu C, Phillip M, et al. Efficacy and safety of dapagliflozin in patients with inadequately controlled type 1 diabetes: The DEPICT-1 52-week study. Diabetes Care. 2018;41:2552-9.

52. Rabizadeh S, Nakhjavani M, Esteghamati A. Cardiovascular and renal benefits of SGLT2 inhibitors: a narrative review. Int J Endocrinol Metab. 2019:17:e84353.

53. MCAllister DA, Read SH, Kerssens J, et al. Incidence of hospitalization for heart failure and case-fatality among
3.25 million people with and without diabetes mellitus. circulation. 2018;138:2774-86.
54. Zelniker TA, Wiviott SD, Raz I, et al. SGLT2 inhibitors for primary and secondary prevention of cardiovascular and renal outcomes in type 2 diabetes: a systemetic review and meta-analysis of cardiovascular outcome trials Lancet. and meta-analysis of cardiovascular outcome trials. Lancet.

(201,

dapagliflozin as an adjunct to insulin on urinary albumin-tocreatinine ratio over 52 weeks in adults with type 1 diabetes Presented at: American Society of Nephrology, Kidney Week San Diego California, 23-28 October 2018.

56. Danne T, Garg S, Peters AL, et al. International consensus on risk management of diabetic ketoacidosis in patients with type 1 diabetes treated with sodium-glucose cotransporter (SGLT) inhibitors. Diabetes Care. 2019;42:1147-54.

57. EMC. Forxiga $5 \mathrm{mg}$ film-coated tablets, SMPC. Available at: 11 August 2020)

58. EMC. Forxiga $5 \mathrm{mg}$ film-coated tablets, Risk Materials. Available at: www.medicines.org.uk/emc/product/2865/rmms (accessed 11 August 2020).

59. Forshcungsinstitut Diabetes. KetoAWARE. Available at: https://fidam.de/ketoaware (accessed 11 August 2020).

60. Yang Y, Chen S, Pan $\mathrm{H}$, at al. Safety and efficacy of SGLT2 inhibitor combining with insulin in subjects with diabetes. Systemic review and meta-analysis of randomized controlled trials. Medicine (Baltimore). 2017;96:21

61. Lind M, Svensson A-M, Kosiborod M, et al. Glycemic control and excess mortality in type 1 diabetes. $N$ Engl I Med. 2014;371:1972-82.

62. Nathan DM; DCCT/EDIC research group. The diabetes control and complications trial/epidemiology of diabetes interventions and complications study at 30 years: overview. Diabetes Care. 2014;37:9-16.

63. Lu J, Tang L, Meng H, et al. Effects of sodium-glucose cotransporter (SGLT) inhibitors in addition to insulin therapy on glucose control and safety outcomes in adults with type 1 diabetes: a meta-analysis of randomized controlled trials. Diabetes Metab Res Rev. 2019;35:e3169.

64. Fellinger $P$, Fuchs $D$, Wolf $P$, et al. Overweight and obesity in type 1 diabetes equal those of the general population. Wein Klin Wochenschr. 2019;131:55-60.

65. Liu W, Huang Z, Zhang X, et al. Urinary C-peptide creatinine ratio as a non-invasive tool for identifying latent autoimmune diabetes in adults (LADA). Diabetes Metab Syndr Obes. 2019:12:2531-7.

66. Pieralice S, Pozzilli P. Latent autoimmune diabetes in adults: A review on clinical implications and management. Diabetes Metab J. 2018;42:451-64.

67. Eriksson JW, Bodegard J, Norhammar A, et al. Cardiovascular complications in type 1 and type 2 diabetes patients show more similarities than differences, 2019. Available at: www.easd.org/virtualmeeting/home.html\#!resources/ cardiovascular-complications-in-type-1-and-type-2-diabetescardiovascular-complications-in-type-1-and-type-2(accessed 11 August 2020). 